\title{
A noval risk assessment method and application for the on-site power operations
}

\author{
Boya Peng ${ }^{1}$, Wei Zhang ${ }^{2}$, Xiangyu Lin ${ }^{3}$, Hao Qiu ${ }^{4}$ \\ Electric Power Research Institute of Guangxi Power Grid Co., Ltd, Nanning, China \\ ${ }^{1}$ Corresponding author

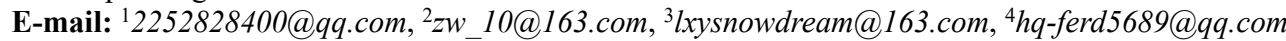

Received 1 April 2021; received in revised form 12 April 2021; accepted 19 April 2021 DOI https://doi.org/10.21595/vp.2021.21973

A) Check for updates

Copyright $₫ 2021$ Boya Peng, et al. This is an open access article distributed under the Creative Commons Attribution License, which permits unrestricted use, distribution, and reproduction in any medium, provided the original work is properly cited.

\begin{abstract}
In order to improve the risk warning and management ability of power industry workers to implement an on-site power operation, this paper proposes a new risk assessment method based on a fuzzy comprehensive assessment method with variable weight. This method begins with selecting the factors related to the risk assessment of on-site power operations. And then, the Analytic Hierarchy Process (AHP) method is used to determine the initial value of the relative weight of the risk assessment factors. Afterward, according to the actual on-site power operation situation, the score value of the risk assessment factors can be obtained. This paper innovatively proposed a variable weight mechanism, which redistributes the relative weight of risk assessment factors according to the scores of risk assessment factors. And through the membership function to determine the fuzzy judgment risk matrix to obtain the risk level of power operations. Finally, take the actual on-site power operation of " $220 \mathrm{kV}$ line protection inspection of a power department" as an example. Comparing the evaluation strategy of this paper with the traditional evaluation strategy, we can concluded that the evaluation strategy of this paper not only can obtain the risk level of electric power operation directly, but also can judge whether there are potential high-risk factors in the electric operation through the degree of medium-risk or high-risk membership.
\end{abstract}

Keywords: risk assessment, on-site power operations, fuzzy comprehensive assessment method, variable weight.

\section{Introduction}

In recent years, accidents in the process of on-site power operations occurred sometimes, causing greater harm to power industry workers and the power grid [1-3]. In order to avoid the hazards and risks in electric power operations, countries in the world have designated risk laws and regulations. Ref [4] introduces some electrical risk-related laws and regulations in Italy, and gives some certain safety management measures. However, these traditional on-site operation risk assessment process mainly depend on the experience of risk assessors to judge, carry out hazard identification and risk grade assessment, make the subjective factors of the risk assessment result are relatively large $[5,6]$.

In this paper, in order to conduct a more scientific and reasonable risk assessment method of on-site power operations, the traditional power operation risk assessment method can be improved from two aspects. On the one hand, with the development of information technology of the electric power industry, a risk assessment factors can be concerned based on big data. That is to say the risk assessment related data can be obtained from various power industry systems, these data always can be divide into several risk assessment factors, which can be divide to personnel abilities, operating methods, equipment status, machinery, equipment and tools, and operating environment and time. Therefore, a multi-dimensional dynamic risk assessment method can be realized. On the other hand, in order to realize a dynamic risk assessment of on-site power operation, a variable weight mechanism and reasonable risk assessment method are concerned. This paper uses the Analytic Hierarchy Process (AHP) to determine the initial value of the weight 
[7]. Moreover, the weight with a higher risk score is modified by the variable weight method. By comparing the score value of actual risk assessment factor of on-site power operation with the set dangerous limit value, the initial value of the weight of the power operation risk assessment factors can be adaptive adjust according to the variable weight mechanism. Last but not least, to obtain the risk assessment grade more reasonably. Fuzzy comprehensive evaluation method can analyze risk assessment grade of the on-site power operations, the factors related to risk assessment can be expressed and evaluated by fuzzy language, and the risk grade of on-site power operation risk assessment can be signified by risk matrix $[8,9]$. The final actual example verifies the rationality of the proposed method, that is to say, the proposed method can complete the objective and accurate risk assessment of the on-site power operation, and lay the foundation for reducing the occurrence of accidents. The contribution of this paper are listed as follows:

1. The variable weight method can make the weight of risk assessment factors more reasonable.

2. In order to get the risk assessment result automatically, the membership function is used.

3. The risk assessment result of electric power operation is actually a fuzzy state. Moreover, according the proposed method, the potential risk factors of power operation can be uptapped according to the distribution of risk membership degree.

\section{Risk assessment factors of on-site electric power operation}

According to the actual electrical operation, a number of risk assessment factors of on-site power operations are specifically selected. In detail, the first level of factors are historical data (A), personnel ability (B), mechanical equipment and tools (C), operation methods (D), power grid equipment status $(\mathrm{E})$, and operating environment and time $(\mathrm{F})$.

\section{On-site power operation risk assessment method based on variable weight fuzzy comprehensive evaluation}

The weight calculation method adopts by this paper a power operation risk assessment factors, which combines the AHP method and the variable weight mechanism. The AHP method determines the initial value of the power operation risk assessment factors weight. At the same time, based on the variable weight mechanism, according to the power operation risk assessment factor. When the actual score value is larger than the set limit value, thus, the possibility of risk be-comes greater.

\subsection{Variable weight mechanism}

\subsubsection{Weight penalty correction based on the barrel effect}

In the power operation risk assessment, when the score value of an actual power operation risk assessment factor is very high and exceeds the set dangerous value, the assessment factors may directly affect the safety level of this on-site power operation, so it is necessary to increase the weight value of the corresponding factor. Assuming that there is a factor score value exceeds the set dangerous value, the subscript of the corresponding factor is set as $A$, the weight correction formula for these dangerous factors can be expressed as:

$\omega_{i}=e^{\omega_{i 0} \frac{\left(x_{i}-x_{d}\right)}{x_{i}}}, \quad i \in A$,

where $\omega_{i}$ is the corrected weight value of the risk assessment factor; $\omega_{i 0}$ is the initial weight value of the risk assessment factor; $x_{i}$ is the current risk assessment factor score value; $x_{d}$ denotes the limit risk value of the risk assessment factor. 


\subsection{Adaptive weight modification of key indicators}

When the score value of an actual power operation risk assessment factor is very high and close to the set dangerous value, and exceed the critical value, the importance of the factor in the power operation risk assessment will increase, so the weight value corresponding to the factor will also become larger. Assuming that there are $b$ assessment factors whose score values are close to the set critical value and the subscript is $B$; the other $c$ evaluation factors whose subscript is set as $C$, and then, the weight correction formula of the risk assessment factor is:

$\omega_{i}=\omega_{i 0}+\omega_{i 0}\left(1-\sum_{k \in B} \omega_{k 0}\right), \quad i \in B$

The weight correction formula for normal evaluation factors score value is as follows:

$\omega_{j}=\omega_{j 0}-\omega_{j 0}\left(1-\sum_{k \in C} \omega_{k 0}\right), j \in C$,

$\sum_{i \in B} \omega_{i}+\sum_{j \in C} \omega_{j}=1$

where $\omega_{i}$ is the corrected weight value of the risk assessment index; $\omega_{i 0}$ is the initial weight of the risk assessment index.

\section{Multi-level fuzzy comprehensive evaluation}

Fuzzy comprehensive evaluation method is a comprehensive evaluation method based on fuzzy mathematics. This method uses the membership theory of fuzzy mathematics to transform qualitative evaluation into quantitative evaluation. Therefore, the fuzzy comprehensive evaluation method can make an overall evaluation of these objects, which restricted by multiple factors.

\subsection{Determination of the risk evaluation collection elements}

As shown in Table 1, it is the on-site power operation risk assessment grades, the corresponding evaluation collection elements are set as $V=\left(v_{1}, v_{2}, v_{3}, v_{4}, v_{5}\right)$ according to power operation risk assessment grades.

Table 1. Evaluation grade

\begin{tabular}{|c|c|}
\hline Evaluation collection elements & Evaluation grade \\
\hline$v_{1}$ & Acceptable risk \\
\hline$v_{2}$ & Low risk \\
\hline$v_{3}$ & Medium risk \\
\hline$v_{4}$ & High risk \\
\hline$v_{5}$ & Very high risk \\
\hline
\end{tabular}

\subsection{Multi-level fuzzy comprehensive evaluation}

(1) The defined power operation risk assessment factors are divided into 6 subsets, according to the first-level factors classification criteria [10], and get:

$$
\left\{\begin{array}{l}
\sum_{i=1}^{6} U_{i}=U, \\
U_{i} \cap U_{j}=\Phi(i \neq j) .
\end{array}\right.
$$


Therefore, the first-level power operation risks assessment factors set is:

$U=\left\{U_{1}, U_{2}, \ldots, U_{6}\right\}$

where $U=\left\{U_{i k}\right\}(i=1,2, \cdots 6 ; k=1,2, \cdots n)$ means that each subset contains $n$ judgment factors.

(2) The score values of $\mathrm{n}$ risk evaluation factors in subset $U_{i}$ can be obtained according to the 5 membership functions, and obtain 6 fuzzy evaluation decision matrices:

$R_{i}=\left[\begin{array}{c}R_{i 1} \\ R_{i 2} \\ \vdots \\ R_{i n}\end{array}\right]=\left[\begin{array}{cccc}r_{11} & r_{12} & \cdots & r_{15} \\ r_{21} & r_{22} & \cdots & r_{25} \\ \vdots & \vdots & \ddots & \vdots \\ r_{n 1} & r_{n 2} & \cdots & r_{n 5}\end{array}\right]$.

If the weight distribution of risk evaluation factors in subset $U_{i}$ is:

$W_{i}=\left[\omega_{i 1}, \omega_{i 2}, \cdots, \omega_{i n}\right]$.

So, the comprehensive evaluation result of subset $U_{i}$ is:

$Y_{i}=W_{i} \circ R_{i}=\left[y_{i 1}, y_{i 2}, y_{i 3}, y_{i 4}, y_{i 5}\right]$

where "o" represents a fuzzy operator, that is multiplying the row and column elements and then summing them. Moreover, the result cannot exceed 1.

(3) Fuzzy comprehensive evaluation method will be made on the 6 subsets $U_{i}(i=1,2, \cdots, 6)$ in the power risk assessment index $U$, and the evaluation decision matrix of the 6 subsets will be constructed as:

$R=\left[\begin{array}{l}Y_{1} \\ Y_{2} \\ Y_{3} \\ Y_{4} \\ Y_{5} \\ Y_{6}\end{array}\right]=\left[\begin{array}{lllll}y_{11} & y_{12} & y_{13} & y_{14} & y_{15} \\ y_{21} & y_{22} & y_{23} & y_{24} & y_{25} \\ y_{31} & y_{32} & y_{33} & y_{34} & y_{35} \\ y_{41} & y_{42} & y_{43} & y_{44} & y_{45} \\ y_{51} & y_{52} & y_{53} & y_{54} & y_{55} \\ y_{61} & y_{62} & y_{63} & y_{64} & y_{65}\end{array}\right]$.

If the weight distribution of the 6 subsets $U_{i}(i=1,2, \cdots, 6)$ in the power risk assessment factor $U$ is:

$W=\left[\omega_{1}, \omega_{2}, \omega_{3}, \omega_{4}, \omega_{5}, \omega_{6}\right]$.

Therefore, the fuzzy comprehensive evaluation result of a certain power operation can be obtained as:

$Y=W \circ R=\left[y_{1}, y_{2}, y_{3}, y_{4}, y_{5}\right]$

(4) According to $y_{r}=\max _{1 \leq j \leq 5}\left\{y_{j}\right\}$ in the vector $Y=\left(y_{1}, y_{2}, y_{3}, y_{4}, y_{5}\right)$ of the fuzzy comprehensive evaluation result, the overall risk assessment result of the electric power operation belongs to the corresponding grade.

\section{Case studies}

In order to evaluate and analysis the effectively of proposed method. Take " $220 \mathrm{kV}$ line protection inspection of a power department" as an example, Apply the risk evaluation strategy of 
electric power operation in this paper and the evaluation strategy in literature [6], respectively. Finally, the method proposed in this paper was compared and verified.

\subsection{Comprehensive evaluation results}

According to the above-mentioned first-level risk assessment index weight value and the first-level risk assessment index evaluation result, the comprehensive evaluation result of this power operation is obtained as:

$Y=W \circ R=\left[\begin{array}{llll}0.5289 & 0.3339 & 0.0963 & 0.04090\end{array}\right]$.

According to the maximum degree principle of membership, the method to determine the risk assessment result of the electric on-site power operation from the fuzzy comprehensive evaluation result vector is: the risk assessment grade of the electric power operation generally belongs to the acceptable risk level. However, the membership degree of medium risk and high risk is not 0 , so this power operation task also has potential risk factors.

\subsection{Adopt the evaluation strategy in the literature}

Literature [6] proposes a power operation evaluation calculation method based on the dynamic risk value of the baseline risk value, in which the calculation formula of the power operation risk value is as follows:

$Y=X+\omega_{1} * A+\omega_{2} * B+\cdots+\omega_{n} * N$,

where $X$ is the baseline risk value; $A, B, \cdots, N$ is the second-level evaluation index; $\omega_{1}, \omega_{2} \cdots \omega_{n}$ is the index weight obtained through the AHP method, and finally the risk level of power operations is determined according to the risk level of a certain power grid. As shown in Table 2, it is the classification standard for the risk level of electric power operations in a certain place.

Table 2. Classification standard of risk grade score for electric power operation

\begin{tabular}{|c|c|}
\hline Evaluation grade & Risk criterion \\
\hline Very high risk & $100 \leq$ risk assessment value \\
\hline High risk & $80 \leq$ risk assessment value $<100$ \\
\hline Medium risk & $60 \leq$ risk assessment value $<80$ \\
\hline Low risk & $40 \leq$ risk assessment value $<60$ \\
\hline Acceptable risk & $20 \leq$ risk assessment value $<40$ \\
\hline
\end{tabular}

According to the score values of the indicators in Table 3 , the baseline risk value is 28 ; the risk values of other first-level indicators are: $2.7,12.3,8.5,14,5.5$, and 7.5; Therefore, using the evaluation strategy in [6], the risk evaluation value of power operation is 38 . According to the classification standard of power operation risk level in Table 5, the final power operation risk level is: "acceptable risk". The risk level is consistent with the results obtained by the evaluation strategy of this article.

\subsection{Comparative analysis of results}

As shown in Fig. 1, according to the power operation task risk assessment results, compare the power operation risk assessment strategy proposed in this paper with the power operation risk assessment strategy in [6]. It is easy to see that the evaluation results in the literature [6] can only obtain the risk score and risk level of the power operation, and are not predictive. In this paper, the risk assessment results of electric power operations are presented in the form of risk membership degree. From the results, it can be seen that the degree of "acceptable risk" of this 
electric operation accounts for 0.5289 , and the degree of "low risk" accounts for 0.3339 . The degree of "medium risk" accounts for 0.0963, and the degree of "high risk" accounts for 0.0409. Therefore, according to the principle of maximum degree of membership degree, it can be obtained that the electric operation belongs to the "acceptable risk" level, However, the membership degree of medium and high risk is not 0 , so it is easy to find that there are potential risk factors for this electric operation, which requires safety supervision personnel to be vigilant.

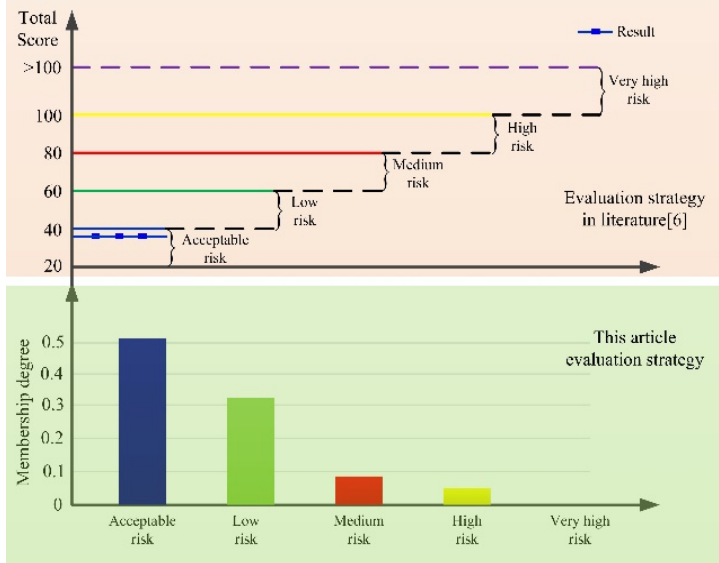

Fig. 1. Comparison of evaluation strategy results in this article and literature [6]

\section{Conclusions}

In this paper, risk assessment related factors are summarized from various power industry systems. And then, the five risk assessment factors together with the fuzzy comprehensive assessment method and variant weight method are innovatively used in this paper. Finally, the risk assessment results show that the risk assessment method can objectively and accurately assess the risk of on-site power operations. It is worth noting that, compared with the traditional evaluation strategy, the evaluation result by proposed method in this paper is given in the form of a risk membership degree. Therefore, not only can the risk assessment result of on-site power operation be obtained accurately, but also can reflect potential risk factors. The proposed method can effectively improve the risk assessment accuracy of on-site power operation in the power industry. Laying foundation for big data-based on-site operation risk assessment, and carrying out dynamic management of on-site operation risks and reducing accidents.

\section{References}

[1] Junzhe L., Lu S., Quansheng Z., Jingjing L. Research on evaluation system of operation risk assessment and application in Henan Power Gird. IEEE PES Asia-Pacific Power and Energy Engineering Conference, Hong Kong, 2014.

[2] Sun Yibin, Ma Taotao, Huang Bingfei, Xu Wei, Yu Bin, Zhu Yingwei Risk assessment of power system secondary devices for power grid operation. China International Conference on Electricity Distribution, Shanghai, 2012.

[3] Zhang Wei, Wu Qiu Li, Deng Yu Rong, Liang Jun Bin, et al. Case-based reasoning adaptive optimization algorithm for power transformer fault diagnosis. Computer Modelling and New Technologies, Vol. 18, Issue 10, 2014, p. 76-81.

[4] Maialetti R. Electrical risks assessment and management at work in Italy. IEEE International Conference on Environment and Electrical Engineering and IEEE Industrial and Commercial Power Systems Europe, Milan, 2017.

[5] Xie M., Yin Y., Lu E., Hu S. Study on human factor risk quantification and evaluation model in power grid dispatching risk assessment. China International Conference on Electricity Distribution (CICED), Xi'an, 2016. 
[6] Wang F., Lin J., Li Y., Yang X. Research on risk assessment method of power industry field operation based on big data. Value Engineering, Vol. 37, Issue 33, 2018, p. 1-4.

[7] Multazam T., Putri R. I., Pujiantara M., Priyadi A., Hery P. M. Wind farm site selection base on fuzzy analytic hierarchy process method; Case study area Nganjuk. International Seminar on Intelligent Technology and Its Applications (ISITIA), Lombok, 2016, p. 545-550.

[8] Wang W., Shen S. Risk assessment model and application for the urban buried gas pipelines. 8th International Conference on Reliability, Maintainability and Safety, Chengdu, 2009, p. 488-492.

[9] Bustince H., Galar M., Bedregal B., Kolesárová A., Mesiar R. A new approach to interval-valued choquet integrals and the problem of ordering in interval-valued fuzzy set applications. IEEE Transactions on Fuzzy Systems, Vol. 21, Issue 6, 2013, p. 1150-1162.

[10] Liu M., et al. Risk assessment of power grid catastrophic accident based on AHP and fuzzy simulation. IEEE International Conference on Applied Superconductivity and Electromagnetic Devices, Beijing, 2013, p. 18-21. 